\section{Commentary: Come on maybe lyse my fire? Cell-free DNA: A potential novel therapeutic target for postcardiopulmonary bypass inflammation}

\author{
Paul Joseph Devlin, MD, MSc, ${ }^{\mathrm{a}}$ and \\ Sunjay Kaushal, $\mathrm{MD}, \mathrm{PhD}^{\mathrm{b}}$
}

Although postoperative mortality in pediatric cardiac surgery has declined significantly, it will remain an important target with higher-risk infants undergoing surgeries and neonates undergoing complete repairs. The mortality hazard is highest during the immediate postoperative period and anticipating poor outcomes may help mitigate adverse events and save lives. ${ }^{1}$ Furthermore, a novel therapeutic agent that targets this early hazard could positively influence the lives of our patients. Among the sources of increased postoperative mortality is the systemic inflammatory response associated with cardiopulmonary bypass. ${ }^{2}$ Mitigating this inflammatory response is an important potential therapeutic target.

Scott and colleagues ${ }^{3}$ from the Medical College of Wisconsin have added to their body of work analyzing the association between nuclear and mitochondrial cell-free DNA and outcomes after cardiac surgery. The release of DNA into the bloodstream from cell apoptosis or cell lysis (hence cell-free) significantly increases with cell and tissue injury. With this prospective observational study of 59 infants who underwent cardiac surgery, the authors have provided early

From the ${ }^{\mathrm{a} D i v i s i o n}$ of Cardiac Surgery, Bluhm Cardiovascular Institute, and ${ }^{\mathrm{b}}$ Division of Cardiac Surgery, Department of Surgery, Ann \& Robert H. Lurie Children's Hospital of Chicago, Northwestern University Feinberg School of Medicine, Chicago, Ill.

Disclosures: The authors reported no conflicts of interest.

The Journal policy requires editors and reviewers to disclose conflicts of interest and to decline handling or reviewing manuscripts for which they may have a conflict of interest. The editors and reviewers of this article have no conflicts of interest.

Received for publication Nov 8, 2021; revisions received Nov 8, 2021; accepted for publication Nov 8, 2021; available ahead of print Nov 12, 2021.

Address for reprints: Paul Joseph Devlin, MD, MSc, Division of Cardiac Surgery, Bluhm Cardiovascular Institute, Northwestern University Feinberg School of Medicine, 676 N Saint Clair St, Suite 730, Chicago, IL 60611 (E-mail: Paul-Devlin@ northwestern.edu).

J Thorac Cardiovasc Surg 2022;164:376-7

$0022-5223 / \$ 36.00$

Copyright (c) 2021 by The American Association for Thoracic Surgery

https://doi.org/10.1016/j.jtcvs.2021.11.015

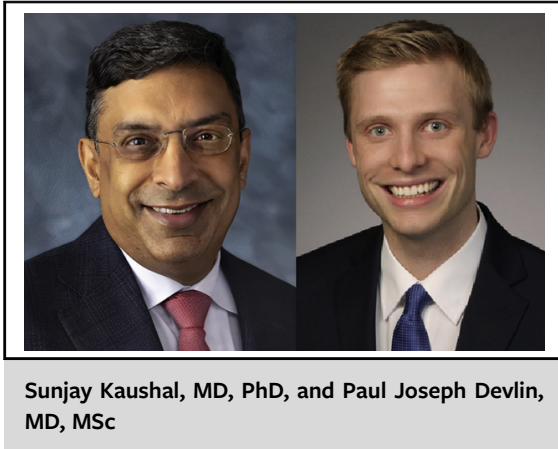

CENTRAL MESSAGE

Cell-free DNA is associated with poor outcomes in infants after cardiac surgery and may serve as a target for future therapeutic agents aimed at reducing postcardiopulmonary bypass inflammation.

data suggesting that persistent elevation in the levels of both nuclear and mitochondrial cell-free DNA is associated with postoperative mortality and prolonged length of stay.

Three infants $(5 \%)$ in the study died during their index hospitalization. All patients underwent testing of nuclear and mitochondrial cell-free DNA at incision, immediately after weaning from cardiopulmonary bypass, and 12 and 24 hours after initiation of cardiopulmonary bypass. In all patients, nuclear cell-free DNA levels increased immediately after cardiopulmonary bypass and for the first 12 hours. However, in the survivors, the levels returned to baseline at later time points, whereas the levels tended to remain elevated in the patients who died. Higher levels of nuclear cell-free DNA were associated with longer cardiopulmonary bypass times, longer crossclamp times, and higher vasoactive inotrope scores during the first 24 hours. The outcome was similar with mitochondrial cell-free DNA. These levels tended to remain elevated postoperatively in the patients who died, although they returned to baseline by 12 hours in most of the survivors, slightly faster than the nuclear cell-free DNA. Mitochondrial cell-free DNA levels were similarly associated with longer cardiopulmonary bypass times and longer crossclamp times.

Seventeen infants $(29 \%)$ required prolonged hospitalization with zero hospital-free days within the first 
30 days after surgery. Levels of both nuclear and mitochondrial cell-free DNA were significantly higher at various time points in these infants. Combined analysis of nuclear and mitochondrial cell-free DNA had high specificity for $(93 \%)$, although low sensitivity $(31 \%)$ for prolonged hospitalization.

Overall, the authors have provided important early data linking nuclear and mitochondrial cell-free DNA levels to adverse outcomes in infants undergoing cardiac surgery. However, one may argue that based on this cohort, the cell-free DNA levels did not provide much prognostic value. Of the 3 infants who died, all received extracorporeal membrane oxygenation (ECMO) postoperatively with subsequent multiorgan failure and likely high vasoactive inotrope scores. It might be that ECMO stimulates more damaging and sustained effects on an inflamed body after cardiopulmonary bypass, which results in higher levels of nuclear and mitochondrial cell-free DNA. Elucidating how the effects of ECMO or cardiopulmonary bypass cause higher levels of nuclear and mitochondrial cell-free DNA will be important to understanding the mechanism of this observation. In our opinion, this research poses significant value in the role of identifying nuclear and mitochondrial cell-free DNA as potential novel therapeutic targets. As the authors have pointed out, cell-free DNA promotes systemic inflammatory response syndrome and its associated vascular tone abnormalities. ${ }^{4}$ Mitigating the systemic inflammatory response in infants after cardiopulmonary bypass through the lysis of cell-free DNA would be a major contribution to the field of pediatric cardiac surgery and could improve postoperative outcomes. Clearly, more data are needed, but this study provides valuable underpinnings for future research.

\section{References}

1. Parikh CR, Greenberg JH, McArthur E, Thiessen-Philbrook H, Everett AD, Wald R, et al. Incidence of ESKD and mortality among children with congenital heart disease after cardiac surgery. Clin J Am Soc Nephrol. 2019;14:1450-7.

2. Kozik DJ, Tweddell JS. Characterizing the inflammatory response to cardiopulmonary bypass in children. Ann Thorac Surg. 2006;81:S2347-54.

3. Scott JP, Tanem JM, Tomita-Mitchell A, Hoffman GM, Niebler RA, Liang HL, et al. Elevated nuclear and mitochondrial cell-free deoxyribonucleic acid measurements are associated with death after infant cardiac surgery. $J$ Thorac Cardiovasc Surg. 2022; 164:367-75.

4. Paunel-Görgülü A, Wacker M, El Aita M, Hasan S. cfDNA correlates with endothelia damage after cardiac surgery with prolonged cardiopulmonary bypass and amplifies NETosis in an intracellular TLR9-independent manner. Sci Rep. 2017;7:17421. 